\title{
Mining Coronal Loops in Solar Images from the SOHO collection
}

\author{
Nurcan Durak, Olfa Nasraoui, Jonatan Gomez, Fabio Gonzalez, Heba Elgazzar, Sofiane Sellah, Carlos \\ Rojas, Joan Schmelz, Jennifer Roames, Kaouther Nasraoui.
}

\begin{abstract}
We present our preliminary findings as part of a new data mining application aiming at the automatic detection of images with coronal loops from one of NASA's solar image databases, known as EIT. Coronal loops are immense arches of hot gas on the surface of the Sun, thought to be jets of hot plasma flowing along in the alleys between the strong coronal magnetic fields. We use various data mining techniques including combining crisp and fuzzy classifiers for automated detection of blocks extracted from EIT solar images. Our data mining and retrieval system helps provide relevant data to astrophysicists who need such data to study the solar corona, and whose work is traditionally hindered by the need to manually sift through thousands of images in order to locate the very few that are useful for further analysis. Our data-driven approach is distinct from related image processing based approaches that cannot scale to large image databases because they rely mostly on semi-automated detection and on heavy and computationally intensive local shape analysis. $^{1234}$
\end{abstract}

\section{INTRODUCTION}

The Coronal Heating Problem [8,9] is one of the longest standing unsolved mysteries in astrophysics, and is essentially concerned with understanding and modeling the exact properties of temperature distribution along coronal loops. The corona is the uppermost level of the Sun's (or another star's) atmosphere, lying immediately above its visible surface. Coronal loops (see Figure 1) are immense arches of hot gas on the surface of the Sun, thought to be jets of hot plasma flowing along in the alleys between the strong coronal magnetic fields. They are visible at X-ray, ultraviolet, and white-light wavelengths, and consist of an arch, extending upward from the photosphere for tens or hundreds of thousands of kilometers. Bright coronal loops, in the form of

\footnotetext{
${ }^{1}$ Nurcan Durak, Olfa Nasraoui, Sofiane Sellah, Heba Elgazzar, and Carlos Rojas are with the Dept. of Computer Science and Engineering, University of Louisville, Louisville, KY 40292, USA.

${ }^{2}$ Fabio Gonzalez and Jonatan Gomez are with Dept. of Computer Engineering \& Computer Systems, Universidad Nacional de Colombia, Bogota, Colombia. This work was done while they were at the Dept. of Computer Science and Engineering, University of Louisville.

${ }^{3}$ Joan Schmelz, Jennifer Roames, and Kaouther Nasraoui are with the Dept. of Physics, University of Memphis, Memphis, TN 38152, USA.

${ }^{4}$ This work is supported by the National Aeronautics and Space Administration under Grant No. AISR-03-0077-0139 issued through the Office of Space Sciences and by the National Science Foundation under Grant IIS-0431128.
}

coronal condensations and bright spots, are common around the time of solar maximum (the sun goes through a cycle of activity every 11 years), while larger faint ones, that last days or weeks, are more typical of the quiet corona, when solar activity is low. The two ends of a loop, known as footprints, lie in regions of the photosphere of opposite magnetic polarity to each other. Coronal loops have been linked to complex phenomena on the sun, such as violent coronal mass ejections, solar flares, and solar storms, that affect the rest of the solar system, by shaking the Earth's magnetic field and power grids, and possibly harming satellites and astronomers in space. Measurements of the temperature distribution along the loop length can be used to support or eliminate various classes of coronal temperature models [7]. In order to make progress, scientific analysis requires data observed by instruments such as EIT, TRACE, and SXT [7]. The combination of EIT, TRACE, and SXT information provides a powerful data set that will yield unprecedented detail on the plasma parameters of a variety of coronal loop structures. The biggest obstacle to completing studies of the solar loop properties has been putting the data set together, so that the subsequent scientific analysis is performed. The search for interesting images (the ones with coronal loops) is by far the most time consuming aspect of this scientific task, amounting to a "search for a needle in a haystack". In this paper, we present an approach to sift through data sets downloaded from online NASA solar image databases and automatically discover the rare but interesting images with solar loops, which are essential in studies of the Coronal Heating Problem. The proposed solar loop mining scheme relies on: (i) Collection and labeling of a sample data set of images coming from both categories (with and without solar loops), and (ii) learning models that can classify a solar image into the correct class. In this paper, we present our preliminary results in mining solar loop images that are located outside the solar disk in images captured by the instrument EIT, aboard the joint NASA/European Space Agency's spacecraft SOHO. The rest of this paper is organized as follows: we discuss our efforts in data preparation in Section 2, then proceed to learning classifiers in Section 3, and present our experimental results in Section 4. Finally, we make 


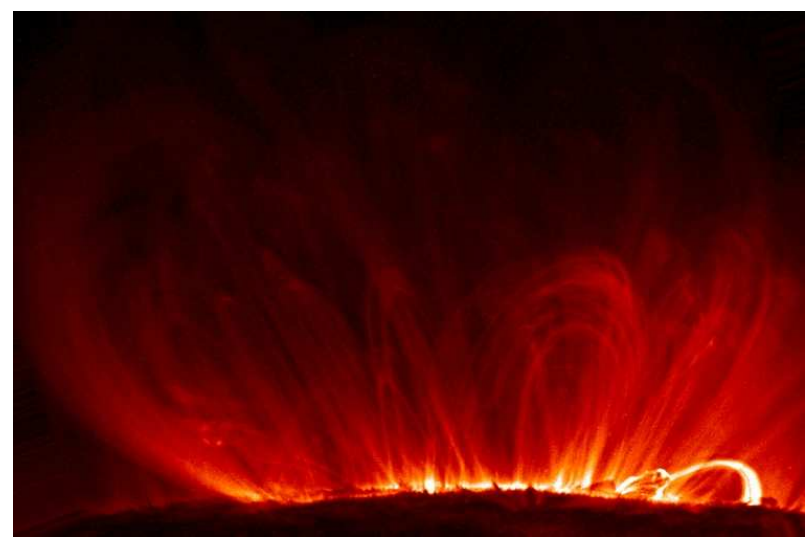

(a)

Fig. 1. A sample TRACE image showing coronal loops on the surface of the sun.

our conclusions, in Section 5.

\section{DATA PREPARATION}

Our initial data (see example in Figure 3 (a)) consists of images in the $171 A^{o}$ Wave length, captured by EIT, an instrument on board of the Solar and Heliospheric Observatory (SOHO), a joint ESA (European Space Agency) and NASA mission to investigate the dynamics of the Sun. Figure 2 shows a rather easy example EIT image with a close up on a coronal loop structure that is well defined. However, most examples have badly defined (weak and broken) loop structures as in Figure 3 (a). We use mostly images in the 171 Angstrom wavelength due to their better quality. Below, we outline the necessary steps needed to prepare the basic data that will be further analyzed and classified. In order to collect the training data, the user has to mark the loop positions in the images, by drawing a rectangular region of interest around each loop. After the marking is done, a set of image blocks, tangent to and outside the solar circle, are extracted from each preprocessed image.

\section{A. Data Transformation}

First, despeckling was applied [4] using a median filter. Then a Sobel edge detector [4] was applied since solar loops are located in regions containing sharp changes in intensity. The edge image is used to compute high level features, while the original intensity image is used to compute low level features. Figure 3 shows a solar image before (a) and after (b) the Sobel edge detection.

\section{B. Block Extraction and Labeling}

A set of image blocks, tangent to the solar circle, are extracted from each preprocessed image. Only blocks

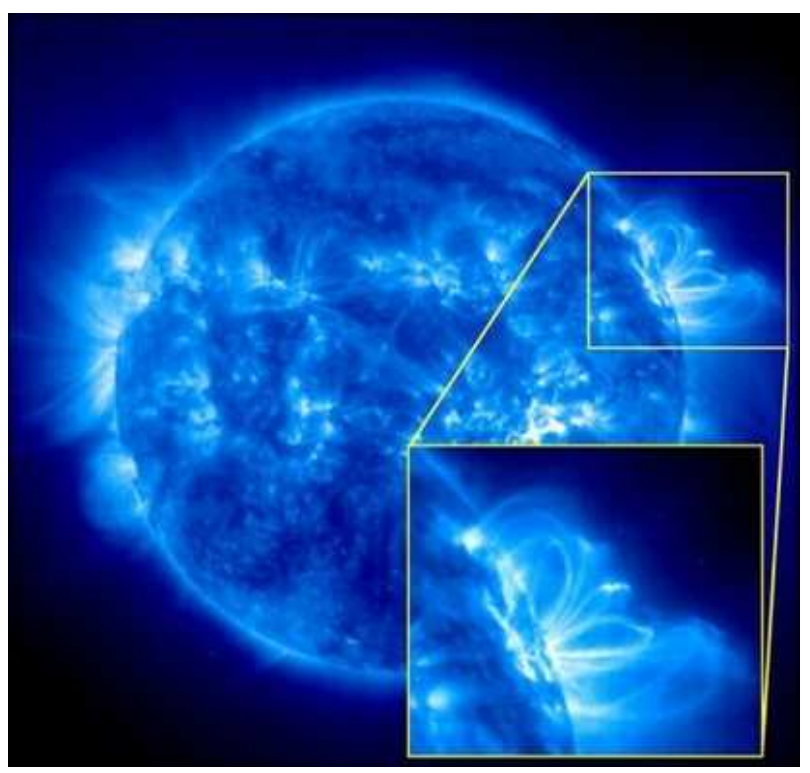

(a)

Fig. 2. An example EIT image with a close up on a coronal loop structure

that are tangent to the solar circumference are extracted. Though we have experimented with several schemes to determine an optimal size based on the marked regions, we have noticed that a fixed size based on trial and error, was actually preferrable especially due to the diverse loop sizes in the data (i.e. there is no one optimal value). After generating several blocks from each image, these blocks are labeled as either containing solar loops (i.e., Loop class), or not (i.e. NO loop class). Doing this labeling manually would require too much effort and time. Thus we resorted to automatic labeling. We consider a block in the Loop class if its intersection with a marked loop region (in the same image) is higher than a certain percentage, that we determined by trial and error (typically around $70 \%$ ).

\section{Feature extraction}

Figure 4 shows two intensity level blocks and the same two blocks after applying edge detection and thresholding. Looking at some of the strongly defined loops, it may seem that some shell clustering methods [19] could detect the elliptically shaped loops. However, our preliminary experiments with EIT images showed that the loops' quality was too poor (see Figure 4(b)) for these techniques to give any reliable detection. Thus we resorted to a supervised learning strategy, where examples of blocks with and without loops are used to build a prediction model that can detect the occurence of loops based on various features. The following low level features [4,5] were extracted from the intensity levels 


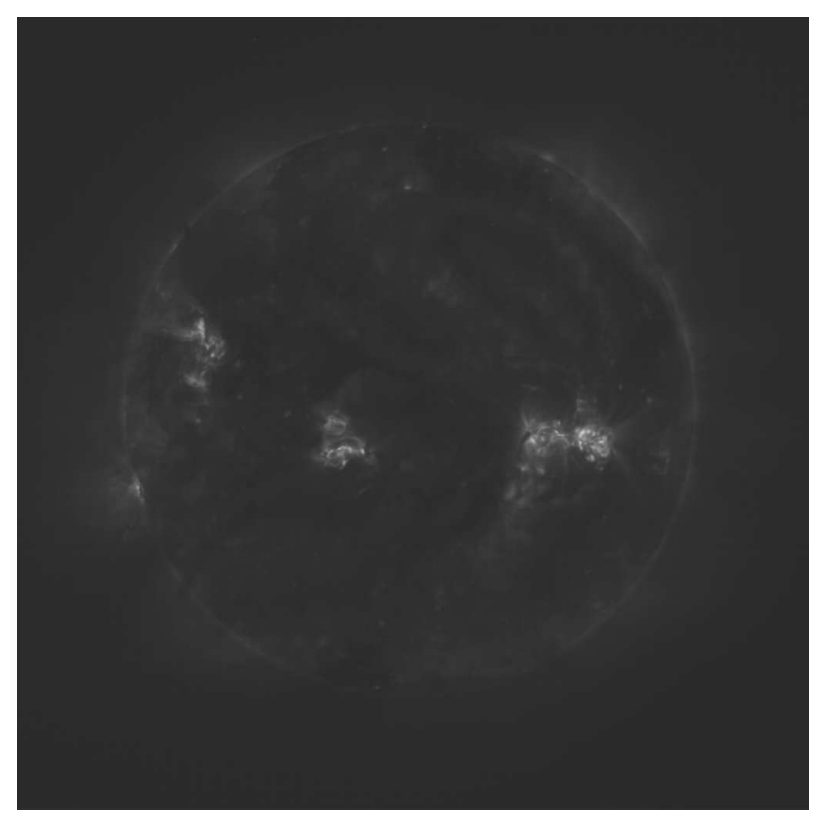

(a)

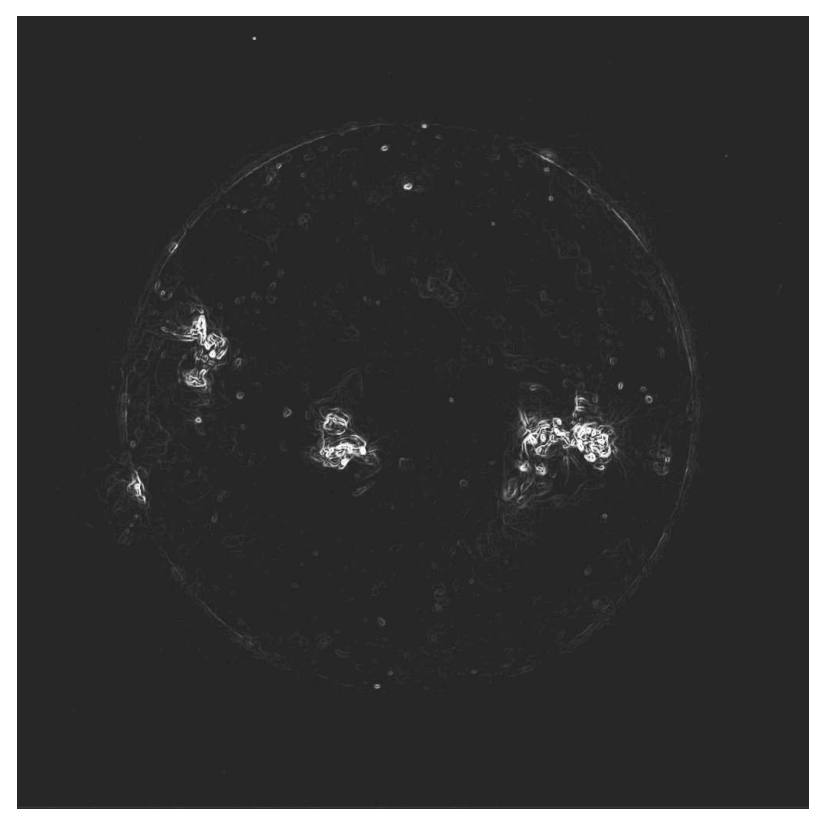

(b)

Fig. 3. Preprocessing to highlight structures: (a) before (used for low level features), and (b) after edge detection (used for high level features). Notice the presence of a loop structure on the left side of the solar disk, just below the equator line.

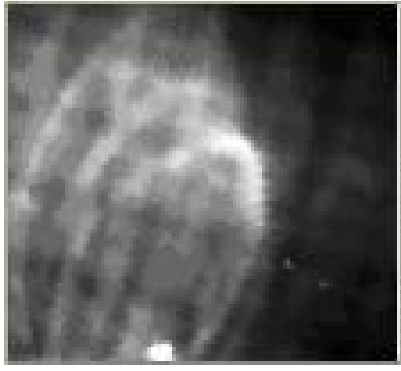

(a)

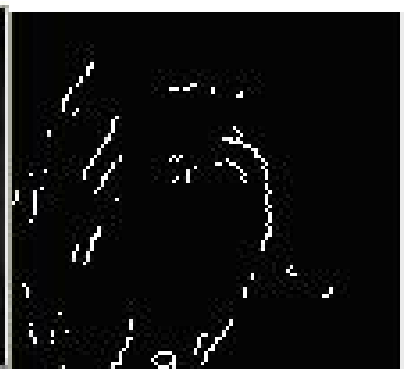

(b)
Fig. 4. (a) Intensity level blocks and (b) binary blocks

of the pixels in each block: Mean, Standard Deviation, Smoothness, Third Moment, Uniformity, and Entropy. In addition, high level features were extracted from the binary edges of the image: Line Direction features (based on angles, as explained below), number of edge pixels, number of line segments, length of the longest line segment (crude estimate of number of edge pixels on estimated lines), and number of edge pixels in each of 4 horizontal bands that make up each block. The latter takes into account location information. Lines were estimated using a crude Hough transform. From our preliminary experiments, the well known Edge Histogram Descriptor features or EHD [13,14] have resulted in worse results than our Line Direction features (based on slope angles of the crudely estimated lines).

For Line Direction features, we have distinguished between different directions of linear segments based on their estimated slopes (from the Hough space), by mapping the ranges of slopes in degrees, as shown below, with angles located outside these intervals considered as non-directional.

- Horizontal: $[0,20]$ and $[160,180]$.

- Vertical: [70,110].

- Diagonal: $[35,55]$ and $[125,145]$.

\section{LEARNING ClASSIFIERS}

Several classification algorithms were compared, including Ripper (a propositional rule learner that learns very concise rules, using Repeated Incremental Pruning to Produce Error Reduction) [16], C4.5 decision trees [20], Multi Layer Perceptron Neural networks (MLP), Support Vector Machines (SVM) [21], Naive Bayes classifiers, and Adaboost [22].

In general, loop blocks can be very diverse in their shape, size, and direction, and in some cases, are very hard to distinguish (even to the untrained Human eye) from other solar phenomena that occurs in the corona, such a coronal mass ejections and solar flares. Figure 5 shows some loop blocks that proved difficult to classify correctly using all the above classifiers. These difficult 


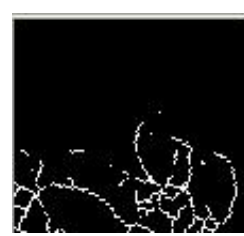

(a)

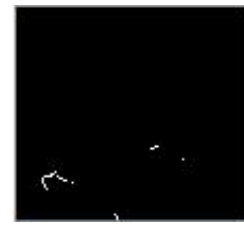

(c)

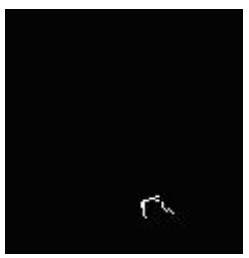

(e)

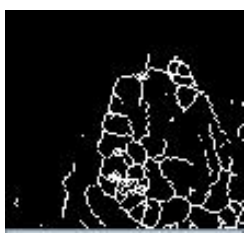

(b)

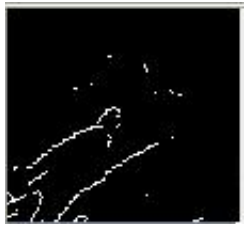

(d)

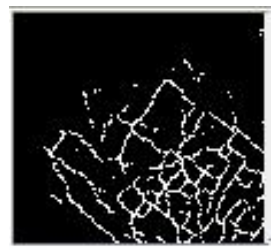

(f)
Fig. 5. Examples of loop blocks that are hard to classify

blocks are generally classified as no-loop by most classifiers because they share a lot of similarity with no-loop blocks. Fuzzy sets [18] can help model these loop blocks better. Figure 6 shows examples of no-loop blocks that are hard to classify correctly.

Modeling both input features and output labels using fuzzy set membership values can help provide a more accurate representation of the ground truth and may help in classification of borderline blocks. In order to construct the fuzzy rules, we have first selected the optimal feature subset from RIPPER's rules and the nodes in the $\mathrm{C} 4.5$ decision tree. These features were: the number of edge pixels, the number of lines segments, the number of edge pixels in the top band, and the length of the longest line. The output of the fuzzy inference system is the block label which can be loop or no-loop. We determined the value ranges of the input parameters from the ranges in RIPPER's rules and the rules derived from the branches of the $\mathrm{C} 4.5$ decision tree. Thus, for each input parameter, we have defined three intervals: low, medium, and high. Some features had an additional value (TooHigh). Their ranges are listed in Table I, and the membership functions are displayed in Figure 7.

Mamdani's fuzzy inference systems [17] consist of ifthen rules in the form "If $x$ is $A$ then $y$ is $B$ ", where $x$ and $y$ are fuzzy variables, and $A$ and $B$ are fuzzy values. We selected the fuzzy rules from the rules that were generated by RIPPER and from short and pure branches

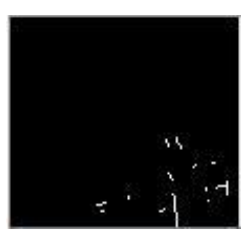

(a)

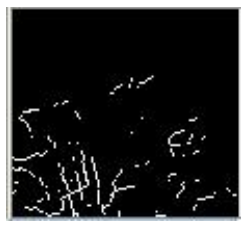

(c)

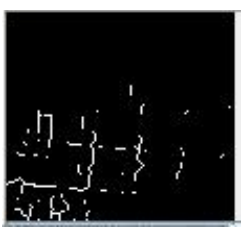

(e)

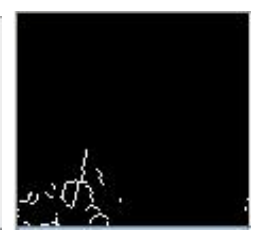

(b)

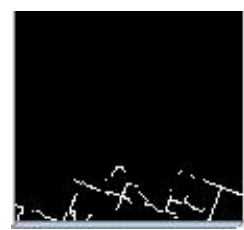

(d)

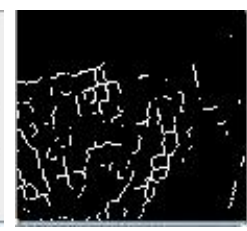

(f)
Fig. 6. Examples of no-loop blocks that are hard to classify

TABLE I

RANGES OF THE FUZZY VALUES FOR THE INPUT FEATURES SELECTED FROM RIPPER'S RULES AND THE OUTPUT LABEL

\begin{tabular}{|l|cc|}
\hline Feature & Intervals & \\
\hline & Low & {$[0-500]$} \\
Number of edge pixels (EdgePixels) & Medium & {$[100-1300]$} \\
& High & {$[700-3000]$} \\
& TooHigh & {$[2500-4500]$} \\
\hline & Low & {$[0-7]$} \\
Number of Line segments (LineSeg) & Medium & {$[4-17]$} \\
& High & {$[14-30]$} \\
& TooHigh & {$[27-35]$} \\
Number of edge pixels in the top & Low & {$[0-40]$} \\
band (EdgePixel-In-TOP-Band) & Medium & {$[20-270]$} \\
\hline \multirow{3}{*}{ Length of longest line segment } & Low & {$[230-400]$} \\
(LineLength) & High & {$[0-70]$} \\
\hline Block label & no loop & {$[0-0.5]$} \\
& loop & {$[0.5-1]$} \\
\hline
\end{tabular}

in the tree generated by $\mathrm{C} 4.5$ decision tree learning. The resulting fuzzy rules are listed in Table II, with the weights that are used to combine all the outputs into the final decision the weights we defined based on the number of data correctly classified in the corresponding RIPPER and C4.5 rules.

\section{EXPERIMENTAL RESUlTS}

We present results for a data set consisting of 103 images, labeled by marking rectangular areas around loop blocks. From these images, 430 loop blocks and 


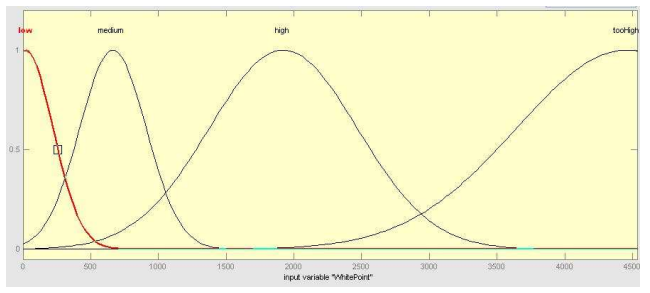

(a)

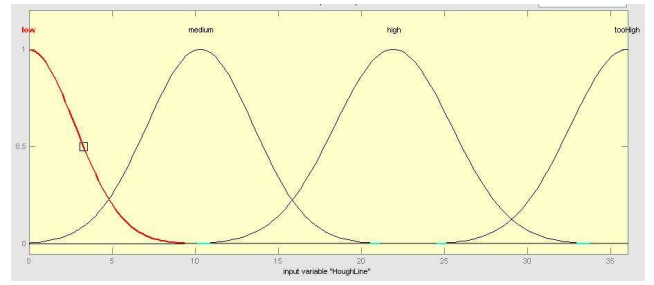

(b)

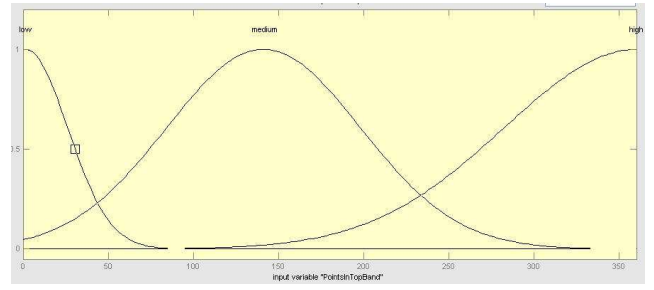

(c)

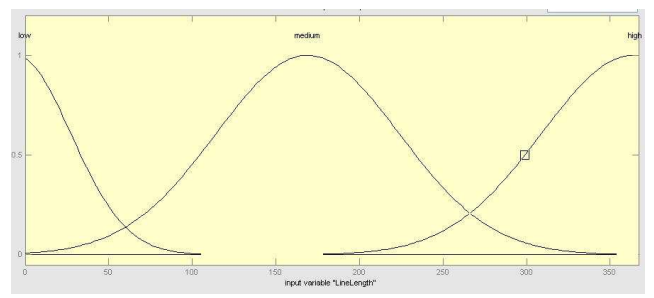

(d)

Fig. 7. Fuzzy membership functions for the input features selected from RIPPER's rules: (a) number of edge pixels, (b) number of lines segments, (c) number of edge pixels in the top band, and (d) length of the longest line segment.

4886 no-loop blocks were extracted. We performed 10fold cross-validation experiments for each of the studied classifiers, implemented in the Weka data mining tool [11] and matlab for the fuzzy classifier, and report the averaged results over 10 independent test sets in Table III. Based on precision and recall in the loop class, the high level features outperformed the low level features. Also, RIPPER resulted in an overall better balance between precision and recall. We have then used RIPPER and C4.5's rules to select the reduced feature set for the inputs to fuzzy inference, and to construct the fuzzy rules. We used Gaussian membership functions with the ranges listed in Table I. The results of the fuzzy classification were increased recall: 0.781 and increased
TABLE II

FUZZY RULES CONSTRUCTED FROM RIPPER AND SELECTED CONCISE AND PURE BRANCHES OF C 4.5

\begin{tabular}{|l|l|}
\hline Condition & $\begin{array}{l}\text { Consequent with } \\
\text { rule weight }\end{array}$ \\
\hline \hline EdgePixels is low & no-loop (0.8) \\
\hline EdgePixels is low AND LineSeg is medium & loop (1) \\
\hline $\begin{array}{l}\text { EdgePixels is low AND EdgePixel-In-TOP- } \\
\text { Band is medium }\end{array}$ & loop (1) \\
\hline $\begin{array}{l}\text { EdgePixels is low EdgePixel-In-TOP-Band } \\
\text { is high }\end{array}$ & loop (1) \\
\hline $\begin{array}{l}\text { EdgePixels is medium AND LineSeg is } \\
\text { medium }\end{array}$ & loop (0.8) \\
\hline EdgePixels is medium AND LineSeg is low & no-loop (0.8) \\
\hline $\begin{array}{l}\text { EdgePixels is medium AND LineSeg is } \\
\text { medium AND LineLength is low }\end{array}$ & no-loop (0.8) \\
\hline $\begin{array}{l}\text { EdgePixels is medium AND LineSeg is } \\
\text { medium AND LineLength is high }\end{array}$ & no-loop (0.8) \\
\hline EdgePixels is high AND LineSeg is high & loop (0.8) \\
\hline $\begin{array}{l}\text { EdgePixels is high AND EdgePixel-In-TOP- } \\
\text { Band is medium }\end{array}$ & loop (0.8) \\
\hline $\begin{array}{l}\text { EdgePixels is high AND EdgePixel-In-TOP- } \\
\text { Band is high }\end{array}$ & loop (0.9) \\
\hline EdgePixels is high AND LineSeg is high & loop (0.8) \\
\hline EdgePixels is high AND LineSeg is tooHigh & no-loop (0.9) \\
\hline EdgePixels is high AND LineSeg is medium & loop (0.8) \\
\hline $\begin{array}{l}\text { EdgePixels is high AND LineSeg is medium } \\
\text { AND EdgePixel-In-TOP-Band is low }\end{array}$ & no-loop (0.8) \\
\hline $\begin{array}{l}\text { EdgePixels is tooHigh AND LineSeg is } \\
\text { medium }\end{array}$ & loop (0.8) \\
\hline EdgePixels is tooHigh AND LineSeg is high & no-loop (0.8) \\
\hline LineSeg is tooHigh & no-loop (1) \\
\hline
\end{tabular}

precision: 0.659, compared to the best result (RIPPER).

\section{CONCLUSIONS}

We have presented our preliminary findings in a new data mining application for retrieval of coronal loop occurrences from NASA's solar observatory Web image databases to support research in astrophysics. Most existing work related to analysis of coronal loops cannot scale to large image databases because they rely mostly on semi-automated detection and on heavy and computationally intensive local shape analysis techniques. We have explored various data mining techniques for classification, and investigated building a fuzzy classifier with rules generated by first selecting the features that appear in the rules generated by the RIPPER classification algorithm, and then constructing rule structures starting with the rule constructs generated by RIPPER and selected short and pure branches from $\mathrm{C} 4.5$ decision trees. The main advantage of fuzzy classifiers is the the richness of representation of the fuzzy sets and in the tolerance of the soft rules that, when combined together, produce better classification than either classifier alone. We are currently extending our system by developing solar cycle-sensitive classifiers that adapt to the specific solar cycle. This is because the appearance of the solar corona and the frequency of coronal loops 
TABLE III

PRECISION AND RECALL (IN THE LOOP CLASS) FOR VARIOUS CLASSIFIERS AND DIFFERENT LEVELS OF FEATURES. BOLD RESULTS ARE FOR BEST TWO CLASSIFIERS BASED ON BOTH PRECISION AND RECALL SIMULTANEOUSLY.

\begin{tabular}{|c|c|c|c|c|}
\hline features' level & low & low & high & high \\
\hline Classifier & Precision & Recall & Precision & Recall \\
\hline AdaBoost & 0.537 & 0.393 & 0.638 & 0.628 \\
\hline SVM & 1 & 0.002 & 0.719 & 0.37 \\
\hline C4.5 & 0.538 & 0.416 & 0.653 & 0.695 \\
\hline RIPPER & 0.54 & 0.36 & 0.641 & 0.763 \\
\hline Naive Bayes & 0.305 & 0.158 & 0.641 & 0.709 \\
\hline MLP & 0.57 & 0.37 & 0.644 & 0.693 \\
\hline $\begin{array}{l}\text { Mamdani } \\
\text { Fuzzy Rules } \\
\text { constructed with } \\
\text { RIPPER and } \\
\text { C4.5 }\end{array}$ & NA & NA & 0.659 & 0.781 \\
\hline
\end{tabular}

varies drastically as the sun cycles from its period of maximum activity (solar maximum) to its period of quiet activity (solar minimum). We are also investigating costsensitive classifiers [1] and constructing richer (and thus more costly to compute) features that may better capture arching structures in the edge images, and intend to add them in the latest stages of a multi-stage classification, where the number of applicable data becomes a small fraction of the whole candidate set.

\section{ACKNOWLEDGMENTS}

This work is supported by the National Aeronautics and Space Administration under Grant No. AISR-030077-0139 issued through the Office of Space Sciences and by the National Science Foundation under Grant IIS0431128.

\section{REFERENCES}

[1] P. Domingos. "MetaCost: A general method for making classifiers cost-sensitive", Proceedings of the Fifth International Conference on Knowledge Discovery and Data Mining, pp. 155-164, 1999.

[2] U. Fayyad, D. Haussler, and P. Storoltz. Mining scientific data. Communications of the ACM, 39(11), pp. 51-57, 1996.

[3] J. Han and M. Kamber. Data Mining: Concepts and Techniques, Morgan Kaufmann Publishers, San Fransisco, CA, 2001.

[4] R.C. Gonzalez and P. Wintz. Digital Image Processing. AddisonWesley, Reading, MA, second edition, 1987.

[5] J.T. Tou and R.C. Gonzalez. Pattern Recognition Principles. Addison-Wesley, Reading, MA, 1974.

[6] T. Mitchell, Machine Learning, McGraw-Hill, Boston, 1997.

[7] J.T. Schmelz, R.T. Scopes, and J.W. Cirtain. Determining Coronal Heating of Plasma Loops Through Differential Emission Measure Analysis. Advances in Space Research, 30 (3), pp. 507-516, 2002.

[8] J.T. Schmelz, J.W. Cirtain, J.E. Beene, H.T. Blevins, D. Ellis, D.A. Medlin, K. Nasraoui, C. Nevels. Coronal loops: Isothermal OR multithermal? Advances in Space Research, 32 (6), pp. 1109-1115, 2003.

[9] J.T. Schmelz. Are Coronal Loops Isothermal?. Astrophysical Journal Letters, 578, L161, 2002.

[10] J.T. Schmelz. Why Stellar Astronomers Should be Interested in the Sun. Advances in Space Research, 32 (6), pp. 895-904, 2003.
[11] I.H. Witten, E. Frank, L. Trigg, M. Hall, G. Holmes, S.J. Cunningham. Weka: Practical machine learning tools and techniques with java implementations. In Proc. ICONIP/ANZIIS/ANNES'99 Int. Workshop: Emerging Knowledge Engineering and ConnectionistBased Info. Systems, pp. 192-196, 1999.

[12] I.H. Witten and E. Frank. Data Mining: Practical machine learning tools with Java implementations. Morgan Kaufmann, San Fransisco, CA, 2000.

[13] D. K. Park, Y. S. Jeon, C. S.Won, S.-J. Park, and S.-J.Yoo, A composite histogram for image retrieval, in Proc. ICME 2000, vol. 1, pp. 355-358, July 2000.

[14] Text of ISO/IEC 15 938-3 Multimedia Content Description Interface Part 3: Visual. Final Committee Draft, ISO/IEC/JTC1/SC29/WG11, Doc. N4062, Mar. 2001.

[15] V. Zharkova, S. Ipson, A. Benkhalil, and S. Zharkov. Feature Recognition in Solar Images. Artif. Intell. Rev. 23, 3 (May. 2005), pp. 209-266, 2005.

[16] W.W. Cohen, Fast Effective Rule Induction, in Machine Learning: Proceedings of the Twelfth International Conference, Lake Taho, California, Morgan Kaufmann, 1995.

[17] E.H. Mamdani and S. Assilian, "An experiment in linguistic synthesis with a fuzzy logic controller," International Journal of ManMachine Studies, Vol. 7, No. 1, pp. 1-13, 1975.

[18] L.A. Zadeh, "Fuzzy sets," Information and Control, Vol. 8, pp. 338-353, 1965

[19] R. Krishnapuram, H. Frigui, and O. Nasraoui: The Fuzzy C Quadric Shell clustering algorithm and the detection of second-degree curves. Pattern Recognition Letters 14(7): 545-552, 1993.

[20] J.R. Quinlan. C4.5: Programs for Machine Learning. Morgan Kaufmann Publishers Inc., 1993.

[21] V. Vapnik, S. Golowich, A. Smola. Support vector method for function approximation, regression estimation, and signal processing, Advances in Neural Information Processing Systems, 1997.

[22] Y. Freund and R.E. Schapire. A decision-theoretic generalization of on-line learning and an application to boosting. Journal of Computer and System Sciences, 55(1), pp. 119139, August 1997. 